\title{
Transnational Engagements: Menstrual Health and Hygiene-Emergence and Future Directions
}

\author{
Edited by Victoria Miller and Inga T. Winkler
}

Over the last decade, menstrual health and hygiene has emerged as a set of efforts to dissolve menstrual-related barriers. As the field continues to grow and receive increasing attention from organizations, donors, governments, and the media, it is important to assess both the risks and rewards of this work. In conversation with one another, representatives from key organizations from around the world reflect on the efforts to address menstrual health and hygiene and what it requires to productively move forward.

Menstrual bygiene management (MHM) bas been closely associated with the water, sanitation and bygiene (WASH) sector. What do you think are the benefits of MHM's close relationship with WASH? Do you also see any potential risks?

Danielle Keiser: Without the initial push of the WASH sector, most of us working on MHM would be lightyears behind where we are now in terms of getting local communities, the media, and the international community to acknowledge the importance of menstrual health and hygiene.

The benefits of the association to WASH are clear: menstrual hygiene involves clean water and soap for cleansing the body and reusable menstrual materials as well as adequate sanitation facilities that are private, safe, and clean and include a way of disposing blood or used materials.

But there are also clear risks of the association with WASH. Menstruation is not just about maintaining cleanliness, it is about a much larger view of the menstrual cycle as a vital sign for health, a source of biological education and an entry point to female health across the lifecycle. Pigeonholing 
any menstrual-related international development work as merely MHM work limits the opportunity to position the reality of the menstrual cycle as a 4-phase cycle and omnipresent process that occurs across many years of women's lives. Menstrual health is so much more than just hygiene management; it's a body of knowledge, a practice, a compass, a yardstick, and an indicator all at the same time.

Neville Okwaro: It is important to look at the strong linkages between menstruation and WASH. We need to consider the safety of various products used to manage menstruation as well as access to safe water to bathe during a girl's or woman's period. Coupled with these WASH-related challenges is the disposal of used sanitary pads and other menstrual products which is a public health concern.

However, reducing menstruation to a WASH issue is narrowing the scope of the human rights that girls and women enjoy. Before even thinking of access to water and sanitation facilities, we need to consider how girls and women can go through their periods each month unimpeded and without disrupting any aspects of their lives. Menstruation is also a multi-sectoral issue that permeates the very essence of life. Menstruation ought to be integrated in all sectors of life-education, gender, water, workforce, reproductive health, and other issues affecting women and girls. Therefore, it cannot be reduced to a WASH issue only; menstruation is a human rights issue.

Ina Jurga: The WASH sector was one of the first sectors to advocate for and integrate MHM, as many organizations work in schools and approached it from the perspective of assuring provision of appropriate facilities for girls coupled with hygiene education. WASH in Schools has many cross-sectorial aspects of providing facilities (usually through WASH sector), and/or health education (through health or education sector).

Jocelyne Alice Ngo Njiki: The association of menstrual hygiene and the WASH sector make it possible to directly confront the government with its responsibilities. In the areas of health or the advancement of women, for example, MHM is one issue among many. In WASH, MHM highlights gender inequalities and provides guidance on adequate infrastructure for MHM that the other sectors could hardly address. Therefore, the WASH sector provides a unique entry point.

Kamini Prakash and Vinod Mishra: We agree that menstruation is a human rights issue. Let's stop looking at women and girls in silos. The focus needs to be on addressing the taboos and transforming the shame and stigma into confidence and pride. This cannot be done if we continue to look at menstruation through sectoral lenses. Even if a woman has access to water and sanitation facilities, she may not be empowered to use them because of the stigma around menstruation. The Bageshwar district in the Himalayan 
state of Uttarakhand is a case in point. Although households have latrines, menstruators are not allowed to use them or the community latrines during their period due to social taboos. Instead, they have to go out in the open to defecate and manage their menstruation.

Gerda Larsson: During the last decade, we have seen increased attention toward menstruation, also at the global level. In September 2018, the U.N. Human Rights Council adopted a resolution adding important new language on water, sanitation, and gender. The resolution makes an unprecedented call to "address the widespread stigma and shame surrounding menstruation and menstrual hygiene by ensuring access to factual information thereon, addressing the negative social norms around the issue and ensuring universal access to hygienic products and gender-sensitive facilities, including disposal options for menstrual products." While this is a tremendous win and the first time a resolution of this magnitude included menstruation, other global strategic documents such as the Sustainable Development Goals (SDGs) and Global Strategy for Women's, Children's and Adolescent Health developed by the World Health Organization (WHO) still lack specific goals and indicators around menstruation. Without such guiding documents and policies there is a risk that governments, organizations, and companies forget to include and think about how menstruation affects their work.

\section{What does it mean to move menstrual bygiene and bealth beyond the WASH sector?}

Danielle Keiser: Our organization, the Menstrual Health Hub, links menstrual health with WASH, but also with Sexual and Reproductive Health and Rights (SRHR), as well as education, work, human rights, economics, et cetera. Because of the omnipresence of the cycle, menstrual health and hygiene are truly intersectional and multi-sectoral issues and should be taken as such and not forced to fit into any one sector. Therefore, the most appropriate context is all contexts in order to mainstream menstrual health. The overarching theme though is aiming for gender equality, since female biology has been a significant cause of women's poverty and oppression. We will only achieve broad-based menstrual health if it is mainstreamed across all areas of life, including business, policy, education, media, and the home.

Julitta Onabanjo: The advocacy and technical contribution from the WASH sector has been and continues to be a critical part of the effort that has ensured today's recognition of MHM as a development and rights issue. That said, at policy and program level most of the attention given to MHM in WASH programs has focused on facilities, hygiene, products, and education for girls in school. This has resulted in girls and women that are out-of-school and marginalized populations, as well as the essential components of community mobilization and engagement of parents, religious leaders, and men 
and boys being for the most part excluded. The prioritization of a lifecycle approach and situating menstruation as a sexual and reproductive health and rights matter allows for a broadening of partnerships beyond the WASH sector. This allows for efforts to ensure that no one is left behind, and addresses other aspects critical to menstrual health, including social norms, stigma, and discrimination. The prioritization of such approaches is key if we are to ensure gender transformation and normalize menstruation.

Gerda Larsson: It means taking on a wider and more holistic perspective. A move toward menstrual health also requires addressing issues such as menstrual pain and gaining a much better understanding of the impacts of menstrual stigma. Through research that The Case for Her funded, we learned that menstruation-related pain can be a primary reason to begin contraception use. By silencing periods or only addressing them in the context of WASH, we risk under-exploring different strategies to address sexual and reproductive health and rights as well as social inclusion.

\section{Do you think there would be more pushback if menstrual health was more closely linked to sexual and reproductive health?}

Jocelyne Alice Ngo Njiki: On the contrary, it would certainly depend on the context, but based on our experience with the sensitization of populations, establishing a link between MHM and sexual and reproductive health would make such programming more attractive. We find a lot of interest in these linkages during our trainings. In fact, using menstrual health as an entry point might help us tackle well-known challenges in the context of sexual and reproductive health. On the other hand, I do think there is a risk that we lose the specificities of menstrual health when it becomes part of the more general sexual and reproductive health discourse.

Kamini Prakash and Vinod Mishra: Even if we wanted to limit our conversations to just MHM, participants invariably ask questions about sexual and reproductive health. If our work is to remain relevant, we need to allow our training sessions to be learner-led. It is difficult to discuss menstruation in isolation of sexual and reproductive health. For example, one of the female participants in Muzaffarnagar in Uttar Pradesh asked us how she could prevent her husband from having sex with her during her period. Instead of limiting the conversation to menstruation in this case, we made it a point to highlight that a woman has a right over her own body and can refuse sex-even when she is not on her period. Trainers should be equipped to talk about these issues—regardless of a possible pushback—because they are fundamental to gender equality. 
Julitta Onabanjo: We know that across the continent, African societies and their views around sexuality and menstruation are diverse, some remaining relatively static and others quickly evolving. While menstruation is embraced by some societies as a rite of passage to womanhood, there are others in which menstruation is veiled in secrecy and conservatism, or in notions of impurity - as is often the case with many other sexual and reproductive health matters. In the twenty-first century, we must recognize and appreciate changing gender roles and identities and what this means to policy and programming. We must also take into consideration traditional and cultural practices that have held us back or that could move us forward. Overall, we need to consider menstruation in the context of healthy sexuality, rights, and choices.

As we normalize menstruation, we must not trivialize the reality of menstrual irregularities and disorders; premenstrual syndrome and premenstrual dysphoric disorders are real and contraceptive choices are linked to menstrual experiences. In many countries, unmet need for family planning remains high, in part due to women's fears or uncertainties about the effect particular contraceptives may have on their menstrual health. This highlights the importance of rethinking product information to enable women to make an informed choice. As we strengthen health systems to be integrated and accessible to all, we must also focus on a competent health workforce that can promote positive menstrual health, identify early menstrual irregularities and prevent unnecessary discomfort, while recognizing more serious menstrual disorders and referral for specialized care.

Do you think an MHM approach that includes all challenges throughout the reproductive lifecycle would provide a more inclusive approach to menstrual bealth? Specifically, how can MHM begin to address the needs of perimenopausal and menopausal women for example?

Ina Jurga: Absolutely. But for our organization, WASH United, it makes sense to start by focusing on adolescent girls: menarche is not only a key signal for entering puberty but also a critical time to inform about menstrual hygiene and share that menstruation is normal. Furthermore, interventions in schools are generally easy to organize. But yes, it is important to be more inclusive: persons with disabilities, transgender/nonbinary people, and also older women have periods and their specific challenges. One entry point for reaching them could be health workers who have the authority and access to reach more diverse populations.

Julitta Onabanjo: Indeed, with the increased focus on MHM and recognition of menstruation as a sexual and reproductive health issue, we must also recognize that menstrual health is broader than the monthly vaginal bleeding episodes. Vaginal bleeding outside menstruation includes, for example, postpartum bleeding, bleeding after a miscarriage, endometriosis, fibroids, and 
different types of cancers that are associated with heavy bleeding. Likewise, we have an opportunity to address the needs of perimenopausal and menopausal women in our policy and programmatic responses, such as through increased investment in research and education and integration of these topics in pre- and in-service training for service providers to improve access to and quality of services.

Within UNFPA, the United Nations sexual and reproductive health and rights agency, work on menstrual health has evolved significantly over the past decade. UNFPA promotes a lifecycle approach to menstrual health, and to sexual and reproductive health in general, with adequate responses to address the different experiences and challenges encountered throughout the reproductive lifecycle, from menarche to menopause.

Neville Okwaro: It is important that girls and women understand how and why menstruation occurs. This can be best explained through the life cycle approach. Understanding sexual and reproductive health will promote an understanding of menstruation and menstrual health. In many communities, sexuality is riddled with myths, taboos, and mystery. It is not to be talked about in the open. These myths and taboos in turn have a direct effect on the community's view of menstruation. Menstruation can be a stepping stone to challenge the myths and taboos around sexuality while sexual and reproductive health education can be integral in demystifying menstruation and challenging the existing myths, taboos, and beliefs around menstruation.

\section{Menstrual hygiene and health programming in the context of international development has experienced a fast rise over the last years. This is encourag- ing, but do you think this rapid growth might pose any risks?}

Ina Jurga: My experience is that once the challenges have been unveiled, backed by a growing evidence base and champions for the cause, many organizations, governments, and individuals realized that menstrual hygiene was a very neglected aspect and that now it must be considered in programming related to women and girls. Among those now paying attention are donors.

Gerda Larsson: Since 2012, The Case For Her has provided catalytic funding to organizations, research institutions, companies, and individuals tackling menstruation as a means to develop as holistic experts across the space, create evidence of what works and advocate for effective solutions. We are excited to see the growth of the space and believe in sharing the evidence and lessons learned we have gathered. We encourage all organizations to contribute to sharing information and new initiatives to seek out this evidence.

Neville Okwaro: I somehow attribute the fast rise of Menstrual Hygiene and Health programming partly to organizations trying to remain relevant. Donor resources have been dwindling and programs that used to 
receive huge chunks of funding cannot access the same enormous funding. Therefore, organizations have learned to mutate and change strategy to attract funding. MHM programming is rather a new field and aligning an organization's strategy with MHM program translates to more funding. That is why most organizations are involved in sanitary pad distribution- to them, products are the core of MHM.

Ina Jurga: Indeed, with MHM being perceived as new and exciting, there is the risk that it will keep attention until another 'hot' topic emerges in a few years. And because it is 'hot' today, it tends to attract organizations to pitch projects, even if they lack a solid base of experience. From a programming perspective, I hope, MHM will be streamlined and considered as a minimum package for any kind of hardware and software intervention in schools, institutions, and workplace. I hope it will move from trendy to mainstreamed.

Neville Okwaro: At the moment, almost all organizations are coming up with their own understanding of MHM and various training tools and manuals have been developed. There are no standardized MHM Manuals, training tools, and guidelines. Therefore, everyone out there believes that the MHM programming they are running is the best. This is slowly eroding the quality of MHM because many are not so clear on what MHM programming should entail.

Julitta Onabanjo: I agree. A challenge caused by the rapid rise in attention given to MHM is that we now see many parallel programs and initiatives, which may not be well-coordinated, and may instead be driven by resources and donor requirements at a particular point in time, with limited geographical scale, and inadequate attention to the diverse and long-term needs of girls, women, and other people who menstruate, who they intend to serve. In addition, MHM is often positioned as a stand-alone issue, with a relatively narrow programmatic focus on increasing product availability and limited integration into other programs. This highlights the need for concerted efforts to ensure government commitment and ownership within a strong policy environment, enhanced partnership building and multi-sectoral coordination, with strong engagement of policy makers, the private sector, civil society organizations (CSOs), media, and people who menstruate. We need to connect those representing a diverse range of sectors such as education, water and sanitation, trade and industry, gender and social development, innovation, and technology. We must be careful not to silo menstrual health in our efforts to bring it to the fore.

Ina Jurga: Another risk is that without solid research, a lot of communication is based on unverified and not sufficiently robust enough quantitative data and simply repeating information on the alleged impact. Yet, studies based on qualitative research reveal that many women and girls report that 
inadequate resources and the sociocultural context including menstrual stigma have an impact on their menstrual experiences, which negatively impact their physical and mental health, education, employment, and social participation. Evaluations of programs seeking to improve women's and girls' lives suggest that interventions can make a difference, but we must be careful to draw on the right data for the right purpose in the right context.

Neville Okwaro: I agree, there is a dearth of data on MHM. For the data we have, we need to ask ourselves, is the data reliable and representative to the extent that it can be used to make inferences about the true situation? If yes, how many practitioners have tailored their programming based on the available data? How many have generated further evidence and used this evidence for decision-making to inform their MHM programs? We have seen zombie statistics (such as one out of ten girls not attending school when menstruating) circulating. To counter these tendencies, we need to make more responsible use of the existing data and acknowledge its limitations, and we need more research that builds a stronger evidence base.

Gerda Larsson: The question of school absenteeism is just part of a much more complex picture that has yet to be made clear. Funding is needed to solidify the research and evidence base past missed days of school and consider, for example, a student's ability to concentrate in class. To date, the scope of measures has been simplified, and we realize that we have been asking the wrong questions.

Jocelyne Alice Ngo Njiki: We need research addressing menstrual hygiene and health in a range of different disciplines including sociology, biology, physiology, medicine, education, and economics. For example, we would like to know what a society stands to lose financially if it disregards MHM in its public policies and its planning and budgeting. Such studies would help elevate the status of our work.

In other sectors, we have seen that efforts often reach the "low-hanging fruit." Addressing the needs of the most visible risks leaving behind marginalized groups. Have you observed this neglect of certain populations in MHM work?

Julitta Onabanjo: With the rapid rise of MHM programming, most of the existing WASH efforts have focused on girls in school, and left out girls and women not in school, as well as other populations such as transgender people and those with disabilities, as well as the critical components of community mobilization and engagement of parents, religious leaders, and men and boys to address social norms, stigma and discrimination, and normalizing menstruation.

Kamini Prakash and Vinod Mishra: We agree, there are many groups whose special needs have not been considered and addressed, such as girls 
and women with disabilities, menopausal women, women in institutions, homeless women, trans men, women living in disaster settings, to name a few. These groups too often remain unseen and unheard. We need to understand their challenges and see how they can be addressed.

It is also important to note that women do not stay within the home but also go out to work and use public spaces. Toilets in worksites, educational institutions communities, public institutions, and public spaces must be designed to take into consideration menstrual hygiene needs.

Danielle Keiser: With the rapid growth of MHM I also see the risk of leaving boys behind. If we continue to frame menstrual health and puberty changes as girls' and women's issues, there will be major consequences for men and boys, who were 'left out' or considered unimportant to involve. Education around menstruation changes everything-for everyone. After all, teaching about menstrual health is a gateway to talking about bodies more generally, and boys are certainly interested in how their bodies work, too.

Neville Okwaro: It is imperative that researchers capture the voices of vulnerable and marginalized individuals and groups. In that context, numbers cannot just be used as a measure of success. Other qualitative and inclusive data need to be generated. Girls and women will continue to menstruate irrespective of their social status and community setting. Inclusive data will promote equity in decision-making and reach out to marginalized and vulnerable individuals.

Finally, recent critics argue that access to menstrual products is too large a priority in menstrual bygiene and bealth programming and advocacy. Do you agree?

Ina Jurga: From the perspective of menstruating women and girls, who cannot afford or access suitable products, receiving products for free or accessing cheaper products does fulfill a need for hygiene and personal well-being every month. If this keeps girls in schools, or women at the workplace-great. But product distribution needs to come with education including how to use, change, clean, and dispose of products correctly, and even more, education for everyone to empower girls/women/boys/men and to remove negative attitudes and restrictions, and that cannot be done by only providing products.

Neville Okwaro: Concentrating on menstrual products is jumping the gun. Menstrual education is more important than distribution of sanitary pads and assuming menstruators know how to use them. It is vital to pass knowledge on all the available options available to manage menstruation and let users choose for themselves. 
Danielle Keiser: People want quick fixes. They want to see that a problem has an easy answer. And pads and other products seemingly provide that answer. Some may think 'No more suffering for "those poor girls" now that they have pads.' But that is akin to putting a band-aid on a gaping wound-it is only going to bring short-term relief because it does not actually engage with the more complex issues of education, poverty, and inequity at the heart of menstrual health as a social issue. So when we address educational needs, we need to think far beyond menstrual hygiene education (often with a focus on product use) and extend to broader aspects of menstrual health literacy.

Gerda Larsson: It is easy to think that products are the silver bullet, but free pads, tampons, cups, and underwear cannot address menstrual stigma. While products definitely play a role, and absorbents can help a person manage their period, it is not a holistic approach. We need educational programs to tackle insufficient knowledge around menstruation, offset stigma surrounding vaginal bleeding, and provide menstruators with tools to make informed choices.

Kamini Prakash and Vinod Mishra: We agree. There is a risk that the rapid rise of menstrual hygiene and health programming in the recent past may lead to quick-fix solutions such as the provision of menstrual pads, without looking at the other interlinked dimensions of menstrual hygiene such as menstrual taboos, gender inequities, and menstrual waste disposal.

All too often, menstrual hygiene is equated only with the use of menstrual pads. Instead it is important to look at the entire MHM value chain-from breaking the silence and looking at inequitable gender norms, spreading awareness on MHM, providing clean and private WASH facilities and menstrual absorbents, and finally providing safe facilities for disposal of menstrual waste. These are interrelated and interdependent aspects in the MHM chain. For instance, we cannot promote menstrual hygiene without breaking the silence and creating awareness.

In that context, we need more data on the health impact of commercial, disposable sanitary pads which use super absorbent polymers, gels, and other chemicals, so that women and girls can make an informed choice about the menstrual absorbents they use. We also need more data on the environmental risks of disposable pads. With an increasing number of adolescent girls preferring to use disposable pads, the environmental concerns are just coming to light. Schools in India have started installing incinerators to dispose of menstrual pads, but they do not necessarily adhere to emission norms of the Central Pollution Control Board and thus, can be hazardous for school children and staff. By promoting disposable pads, we may be solving the problem of menstrual hygiene but we may also be creating new challenges due to unsafe menstrual waste disposal. 
You all mention the importance of education, but mostly in relation to the use of products. What kind of education do we need to ensure menstrual bealth comprebensively, combat the stigma around menstruation, and enable menstruators to take informed decisions not just in relation to materials but any aspect of menstruation?

Danielle Keiser: The kind of education that is needed is the kind that does not feel like education, but urgency of biological reality and absolute common sense. Separating this from culture is the hard task. Good education starts with the first contact point anyone has with learning about human reproductive biology. Our bodies are not gross and menstruation, while sometimes unpleasant, is natural, normal, and necessary for human life to continue existing as we know it.

Ina Jurga: Our objective has always been to achieve scale and thus the design of our training sessions and educational materials has evolved from providing comprehensive MHM education to offering a very compact, easy-to-use, and low-cost guide (which can also serve as an introduction to more comprehensive or additional materials). Many teachers lack both capacity and comfort to teach about MHM, so we wanted to create something that is very simple, yet covers the basics. Furthermore, and maybe most important, we focus on changing attitudes and empowering girls so they don't feel lonely with their periods and feel empowered to ask questions. The next steps for us will be to explore how we can sustain support and communication beyond the initial training, as well as how to include boys as well as mothers who are often left out, even though they are key informants.

Vinod Mishra and Kamini Prakash: It's not enough to educate menstruators alone when most of the challenges come from the community elders, religious leaders, mothers, and male family members. Mothers, for example, are a primary source of information about menstruation for adolescent girls. Research has shown that $70 \%$ mothers in India consider menstruation as dirty and polluting. Little wonder then that girls grow up observing social taboos which are often harmful and prevent girls from realizing their true potential. We therefore need to extend educational initiatives beyond girls in schools.

At WSSCC, we have started focusing on making our trainings more inclusive. In keeping with the SDG principle of Leave No One Behind, we have developed MHM materials for menstruators with visual and hearing impairments. For example, As We Grow Up: About Menstruation is an instructional video on MHM in Indian Sign Language. We have engaged intensively with the disability sector in India, especially with organizations that represent the visually impaired. To date, we have trained over 70 organizations across India on MHM. 
Julitta Onabanjo: Menstruation impacts educational outcomes and the education system can be an impactful channel to promote and ensure menstrual and other sexual and reproductive health well-being. The importance of access to comprehensive sexuality education and health services becomes vital conduits to ensuring menstrual literacy and normalization. It is also vital to guarantee self-esteem, self-worth, and empowerment that can influence other aspects of sexuality, reproduction as well as general decision-making in life. Menstrual literacy therefore should ideally happen before menarche and move beyond the schoolgirl to include all girls, women, and people who menstruate as well as parents, teachers, community leaders, and other influencers, such as traditional and social media to change the public discourse.

One of the challenges mentioned earlier is the fragmentation between different organizations and approaches. Do you see any initiatives to counter that?

Kamini Prakash and Vinod Mishra: Yes, the coming together of organizations and individuals working on MHM is a sign of progress. In India, the Menstrual Health Alliance India (MHAI) aims to engage with government, civil society, media, and corporations to use evidence-based research to raise awareness and promote inter-sectoral action on MHM. In the last year it has convened a media workshop, given technical recommendations to the government on the Goods and Services tax (GST) on menstrual pads, developed a policy brief and resource book on menstrual waste disposal for the Ministry of Drinking Water and Sanitation and also engaged with Bureau of Indian Standards to develop standards for reusable cloth pads.

Julitta Onabanjo: There are similar developments in the African context. To support efforts to strengthen coordination of existing MHM programs in Africa, UNFPA cohosted the first-ever African Symposium on Menstrual Health Management in Johannesburg, South Africa in May 2018. The event identified knowledge management and information sharing as critical elements of an effective response to menstrual health management in Africa, which led to the establishment of the African Coalition for Menstrual Health Management. The Coalition aims to strengthen advocacy for multi-sectoral policy making, support efforts to fill in existing research gaps, develop product standards and ensure constant and regular availability and diversification of menstrual programs, and products and supplies. The Coalition also supports efforts to ensure that there is proper policy and program guidance to integrate MHM across all relevant sectors.

Given the developments on MHM over the last 5 years and the risks you identified, what do you think needs to happen to strengthen comprehensive programming on menstrual bealth? 
Danielle Keiser: Talking about menstruation openly is the entry point to strengthening comprehensive programming on menstrual health. This will be reflected in a thriving ecosystem of powerful players beyond the development sector who say and do something about it in their everyday work. Investors can fund menstrual health, as it is the foundation for future investments that address female health across the reproductive journey. Policy makers and public servants can advocate for gender-smart policies that look at equity, access, and safety of period products and WASH facilities. Educators can integrate menstrual health in their health, education, and life skills curriculums. Mothers and fathers can prepare themselves by accessing helpful suggestions for guiding their daughters and sons into adulthood.

Neville Okwaro: There is a need to define what constitutes a menstrual health program. It should be holistic and not just address one aspect. It should start from access and move to the provision of practical information which will demystify menstruation including information about menstrual management options and how to dispose of the various materials and products.

Julitta Onabanjo: The need for MHM standards, regulations, unified frameworks, and effective coordination mechanisms cannot be overemphasized. Our point of advocacy is for MHM to be integrated across sectors and areas to ensure that the focus on MHM is expanded from just being about WASH or product availability as a stand-alone initiative, to a more integrated, cross-sectoral policy, and programmatic issue involving adolescents and women's sexual and reproductive health, education and services, and water and sanitation and waste disposal in a broad range of contexts: schools, workplaces, humanitarian settings, and more. 
Open Access This chapter is licensed under the terms of the Creative Commons Attribution 4.0 International License (http://creativecommons.org/licenses/ by $/ 4.0 /)$, which permits use, sharing, adaptation, distribution and reproduction in any medium or format, as long as you give appropriate credit to the original author(s) and the source, provide a link to the Creative Commons license and indicate if changes were made.

The images or other third party material in this chapter are included in the chapter's Creative Commons license, unless indicated otherwise in a credit line to the material. If material is not included in the chapter's Creative Commons license and your intended use is not permitted by statutory regulation or exceeds the permitted use, you will need to obtain permission directly from the copyright holder. 